\title{
New Theoretical Bounds of Visibility Representation of Plane Graphs*
}

\author{
Huaming Zhang and Xin He \\ Department of Computer Science and Engineering, \\ SUNY at Buffalo, Buffalo, NY, 14260, USA
}

\begin{abstract}
In a visibility representation (VR for short) of a plane graph $G$, each vertex of $G$ is represented by a horizontal line segment such that the line segments representing any two adjacent vertices of $G$ are joined by a vertical line segment. Rosenstiehl and Tarjan [6], Tamassia and Tollis [7] independently gave linear time VR algorithms for 2-connected plane graph. Afterwards, one of the main concerns for VR is the size of VR. In this paper, we prove that any plane graph $G$ has a VR with height bounded by $\left\lfloor\frac{5 n}{6}\right\rfloor$. This improves the previously known bound $\left\lceil\frac{15 n}{16}\right\rceil$. We also construct a plane graph $G$ with $n$ vertices where any VR of $G$ require a size of $\left(\left\lfloor\frac{2 n}{3}\right\rfloor\right) \times\left(\left\lfloor\frac{4 n}{3}\right\rfloor-3\right)$. Our result provides an answer to Kant's open question about whether there exists a plane graph $G$ such that all of its VR require width greater that $c n$, where $c>1$.
\end{abstract}

\section{Introduction}

The first simple linear time VR algorithm was given in $[6,7]$ for a 2-connected plane graph $G$. One of the main concerns afterwards for VR is the size of the VR, i.e., the height and width of VR. Some work has been done to reduce the size of VR. We summarize related previous results in the following table:

\begin{tabular}{|l|l|c|}
\hline \hline References & \multicolumn{1}{|c|}{ Plane graph $G$} & 4-Connected plane graph $G$ \\
\hline$[6,7]$ & Width of VR $\leq(2 n-5)$ & Height of VR $\leq(n-1)$ \\
\hline$[2]$ & Width of VR $\leq\left\lfloor\frac{3 n-6}{2}\right\rfloor$ & \\
\hline$[5]$ & Width of $\mathrm{VR} \leq\left\lfloor\frac{22 n-42}{15}\right\rfloor$ & \\
\hline$[3]$ & & Width of VR $\leq(n-1)$ \\
\hline$[8]$ & Height of VR $\leq\left\lceil\frac{15 n}{16}\right\rceil$ & \\
\hline$[9]$ & Width of VR $\leq\left\lfloor\frac{13 n-24}{9}\right\rfloor$ & Height of VR $\leq\left\lceil\frac{3 n}{4}\right\rceil$ \\
\hline \hline
\end{tabular}

In this paper, we obtain the following main results:

(1) We prove that every plane graph $G$ has a VR with height bounded by $\left\lfloor\frac{5 n}{6}\right\rfloor$, which can be obtained in linear time.

\footnotetext{
* Research supported in part by NSF Grant CCR-0309953.
} 
(2) We give a plane graph $G$ with $n$ vertices such that any VR of $G$ require a size of $\left(\left\lfloor\frac{2 n}{3}\right\rfloor\right) \times\left(\left\lfloor\frac{4 n}{3}\right\rfloor-3\right)$. This answers Kant's open question about whether there exists a plane graph $G$ such that all of its VR require width greater that $c n$, where $c>1[2]$.

\section{Preliminaries}

In this section, we give definitions and preliminary results. We abbreviate the words "counterclockwise" and "clockwise" as ccw and cw respectively.

An orientation of a graph $G$ is a digraph obtained from $G$ by assigning a direction to each edge of $G$. We will use $G$ to denote both the resulting digraph and the underlying undirected graph unless otherwise specified. For a 2-connected plane graph $G$ and an exterior edge $(s, t)$, an orientation of $G$ is called an storientation if the resulting digraph is acyclic with $s$ as the only source and $t$ as the only sink. Note that, for every face $f$ of $G$, its boundary cycle consists of two directed paths. The path on its left (right, resp.) side is called the left (right, resp.) path of $f$. There is exactly one source (sink, resp.) vertex on the boundary of $f$, it is called the source (sink, resp.) of $f$.

Let $G$ be a 2-connected plane graph and $(s, t)$ an exterior edge. An stnumbering of $G$ is a one-to-one mapping $\xi: V \rightarrow\{1,2, \cdots, n\}$, such that $\xi(s)=1, \xi(t)=n$, and each vertex $v \neq s, t$ has two neighbors $u, w$ with $\xi(u)<\xi(v)<\xi(w)$, where $u$ (w, resp.) is called a smaller neighbor (bigger neighbor, resp.) of $v$. Lempel et. al. [4] showed that for every 2-connected plane graph $G$ and an exterior edge $(s, t)$, there exists an st-numbering. Given an stnumbering $\xi$ of $G$, we can orient $G$ by directing each edge in $E$ from its lower numbered end vertex to its higher numbered end vertex. The resulting orientation is called the orientation derived from $\xi$ which is an st-orientation of $G$. On the other hand, if $G=(V, E)$ has an st-orientation $\mathcal{O}$, we can define an 1-1 mapping $\xi: V \rightarrow\{1, \cdots, n\}$ by topological sort. Thus, we can interchangeably use the term an st-numbering of $G$ and the term an st-orientation of $G$, where each edge of $G$ is directed accordingly. The following lemma was given in $[6,7]$ :

Lemma 1. Let $G$ be a 2-connected plane graph. Let $\mathcal{O}$ be an st-orientation of $G$. A VR of $G$ can be obtained from $\mathcal{O}$ in linear time. The height of the VR is the length of the longest directed path in $\mathcal{O}$.

Let $G$ be a plane triangulation, $v_{1}, v_{2}, \cdots, v_{n}$ an ordering of the vertices of $G$ where $v_{1}, v_{2}, v_{n}$ are the three exterior vertices of $G$ in ccw order. Let $G_{k}$ be the subgraph of $G$ induced by $v_{1}, v_{2}, \cdots, v_{k}$ and $H_{k}$ the exterior face of $G_{k}$. Let $G-G_{k}$ be the subgraph of $G$ obtained by removing $v_{1}, v_{2}, \cdots, v_{k}$.

Definition 1. [1] An ordering $v_{1}, \cdots, v_{n}$ of a plane triangulation $G$ is canonical if the following hold for every $k=3, \cdots, n$ :

1. $G_{k}$ is 2-connected, and its exterior face $H_{k}$ is a cycle containing the edge $\left(v_{1}, v_{2}\right)$. 
2. The vertex $v_{k}$ is on the exterior face of $G_{k}$, and its neighbors in $G_{k-1}$ form a subinterval of the path $H_{k-1}-\left(v_{1}, v_{2}\right)$ with at least two vertices. Furthermore, if $k<n, v_{k}$ has at least one neighbor in $G-G_{k}$. (Note that the case $k=3$ is degenerated, and $H_{2}-\left(v_{1}, v_{2}\right)$ is regarded as the edge $\left(v_{1}, v_{2}\right)$ itself.)

A canonical ordering of $G$ can be viewed as an order in which $G$ is reconstructed from a single edge $\left(v_{1}, v_{2}\right)$ step by step. At step $k$, when $v_{k}$ is added to construct $G_{k}$, let $c_{l},, \cdots, c_{r}$ be the lower ordered neighbors of $v_{k}$ from left to right on the exterior face of $G_{k-1}$. We call $\left(v_{k}, c_{l}\right)$ the left edge of $v_{k},\left(v_{k}, c_{r}\right)$ the right edge of $v_{k}$, and the edges $\left(c_{p}, v_{k}\right)$ with $l<p<r$ the internal edges of $v_{k}$. The collection $T$ of the left edges of the vertices $v_{j}$ for $3 \leq j \leq n$ plus the edge $\left(v_{1}, v_{2}\right)$ is a spanning tree of $G$ and is called a canonical ordering tree of $G$ [1].

\section{Compact Visibility Representation of Plane Graphs}

Let $G$ be a plane triangulation with $n$ vertices, $v_{1}, v_{2}, v_{n}$ be its exterior vertices in ccw order. Let $T$ be a canonical ordering tree of $G$ rooted at $v_{n}$ with at least $\left\lceil\frac{n+1}{2}\right\rceil$ leaves [8]. We will construct the st-numbering $\xi_{T}$. (see Figure 1 for an illustration. Only part of the tree is drawn. Tree edges are drawn in solid lines, non-tree edges are drawn in dotted lines, dashed lines represent a path in the tree.)

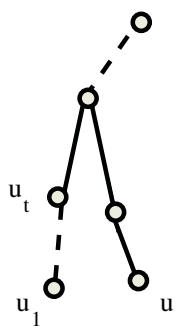

(a)

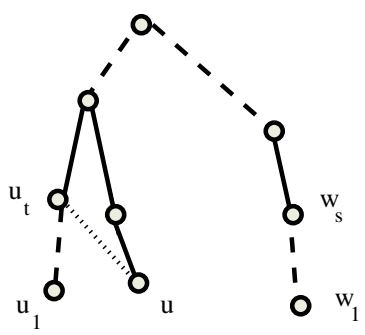

(b)

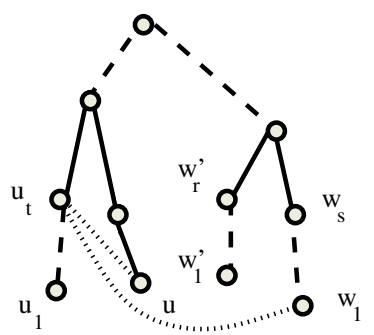

(c)

Fig. 1. (a) No edge between $u_{t}$ and $u$. (b) An edge between $u_{t}$ and $u$, no edge between $u_{t}$ and $w_{1}$. (c) Edges between $u_{t}$ and $u, u_{t}$ and $w_{1}$. No edge between $u_{1}$ and $w_{r}^{\prime}$.

Traveling from the leftmost unassigned leaf of $T$ by ccw postordering with respect to $T$. (The first visited vertex is $v_{2}$ ). Assume we begin from the leaf $u_{1}$, continue to $u_{1}, u_{2}, \cdots, u_{t}$, then we reach the next leaf $u$. There are three cases to be considered for each step:

Case 1: There is no edge between $u_{t}$ and $u$. We then assign the remaining numbers from $1,2, \cdots, n$ to $u_{1}, u_{2}, \cdots, u_{t}$ by ccw postordering with respect to $T$, continue to leaf $u$, continue on, stop before a new leaf encountered. Continue our assignment if there are leaves left unassigned. See Figure 1 (a).

Case 2: There is an edge between $u_{t}$ and $u$, but no edge between $u_{t}$ and $w_{1}$, where $w_{1}$ is the rightmost unassigned leaf of $T$. We then assign the remaining 
numbers from $1,2, \cdots, n$ to $u_{1}, u_{2}, \cdots, u_{t}$ by ccw postordering. Then jump to leaf $w_{1}$, continue on by $\mathbf{c w}$ postordering, stop before a new leaf encountered. Continue our assignment if there are leaves left unassigned. See Figure 1 (b).

Case 3: There is an edge between $u_{t}$ and $u$, and an edge between $u_{t}$ and $w_{1}$. Then Starting from the leaf $w_{1}$, assign the remaining numbers to $T$ by $\mathbf{c w}$ postordering with respect to $T$. Assume that vertices assigned are $w_{1}, w_{2}, \cdots, w_{s}$, then to next leaf, denote it by $w_{1}^{\prime}$, keep assign numbers to $w_{1}^{\prime}, w_{2}^{\prime}, \cdots, w_{r}^{\prime}$ until a new leaf encountered. Jump back to $u_{1}$, keep assign numbers to $u_{1}, u_{2}, \cdots, u_{t}$ by ccw postordering with respect to $T$. Stop before a new leaf encountered. Continue our assignment if there are leaves left unassigned. See Figure 1 (c).

There are at most one or two leaves left at last, then assign remaining numbers to them by ccw postordering. (or $\mathbf{c w}$ postordering). Figure 2 shows such a construction.

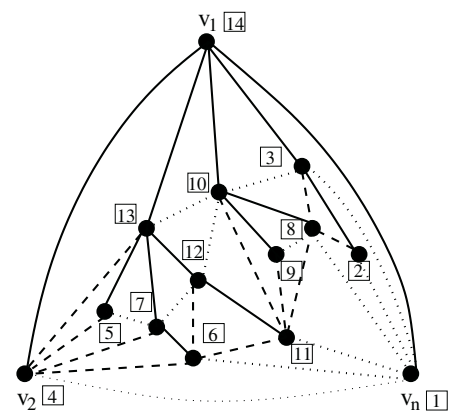

Fig. 2. A plane triangulation $G$, a canonical ordering tree $T$ of $G$ (drawn in solid lines). The $s t$-numbering $\xi_{T}$ of $G$ constructed from $T$ as described above.

Observe that, for each step above, at least one vertex has to be bypassed by any directed path. We have the following:

Theorem 1. Let $G$ be a plane triangulation with $n$ vertices, $T$ a canonical ordering of $G$ with at least $\left\lceil\frac{n+1}{2}\right\rceil$ leaves. Let $\xi_{T}$ be constructed as above. Then:

1. $\xi_{T}$ is an st-numbering of $G$. The longest directed path of $G$ according to $\xi_{T}$ is bounded by $\left\lfloor\frac{5 n}{6}\right\rfloor$.

2. G has a VR with the same exterior face with height bounded by $\left\lfloor\frac{5 n}{6}\right\rfloor$, which can be obtained in linear time.

Next, we have the following theorem regarding the lower bound of VR:

Theorem 2. There is an n-vertex plane triangulation $G$ such that any $V R$ of $G$ with the same exterior face requires a size of $\left(\left\lfloor\frac{2 n}{3}\right\rfloor\right) \times\left(\left\lfloor\frac{4 n}{3}\right\rfloor-3\right)$.

Proof. Suppose that $G_{k}$ is the graph of $k$ nested triangles with $n=3 k$ vertices as shown in Figure 3 (a). We want to show that any VR of $G_{k}$ requires a grid size of $\left(\left\lfloor\frac{2 n}{3}\right\rfloor\right) \times\left(\left\lfloor\frac{4 n}{3}\right\rfloor-3\right)=2 k \times(4 k-3)$. 


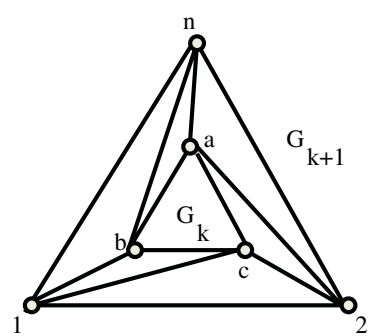

(a)

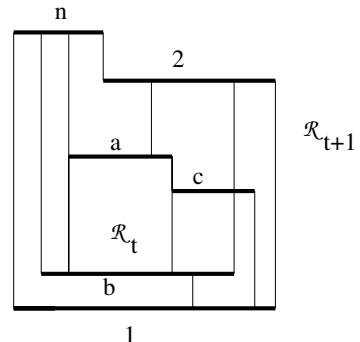

(b)

Fig. 3. (a) Nested triangles, (b) VR of nested triangles.

First, we want to use induction on $k$ to prove the height bound. When $k=1$, it is true. Suppose that it is true for $k=t$. Now, consider $k=t+1$ :

Given any VR $\mathcal{R}_{t+1}$ of $G_{t+1}$, by removing the horizontal lines segments representing the exterior vertices of $G_{t+1}$ and the vertical line segments representing the edges adjacent to the exterior vertices of $G_{t+1}$, the resulting representation is a VR of $G_{t}$, denoted by $\mathcal{R}_{t}$. Applying induction hypothesis, the height of $\mathcal{R}_{t}$ is at least $2 t$.

Observe the topmost and the lowest horizontal line segments in $\mathcal{R}_{t+1}$, they have to represent the exterior vertices of $G_{t+1}$. Thus, the height of $\mathcal{R}_{t+1}$ is at least 2 plus the height of $\mathcal{R}_{t}$, which is at least $2(t+1)$. (See Figure $3(\mathrm{~b})$ ) This finishes the induction for the height bound.

Next, we want to prove the width bound. Given any $\operatorname{VR} \mathcal{R}_{k}$ of $G_{k}$. It is easy to see that we can obtain an st-numbering $\xi$ of $G_{k}$ from $\mathcal{R}_{k}$. We assign numbers from $1,2, \cdots, n$ to the vertices of $G_{k}$ such that the lower its corresponding horizontal line segment in $\mathcal{R}_{k}$ is, the smaller its assigned number is. (If two or more vertices have the same level of horizontal line segments, then arbitrarily assign consecutive numbers to them.) Denote the vertices by $v_{1}, v_{2}, \cdots, v_{n=3 k}$, where $\xi\left(v_{i}\right)=i$. Without loss of generality, we may assume that the vertical line segment representing the edge $\left(v_{1}, v_{n=3 k}\right)$ is the leftmost vertical line in $\mathcal{R}_{k}$. $G_{k}$ can be directed according to $\xi$. And $\xi$ induces its dual st-orientation $\xi^{*}$ of $R_{k}^{*}$.

Claim: The width of the $\operatorname{VR} \mathcal{R}_{k}$ is greater or equal to the length of the longest directed paths in $\xi^{*}$ of $G_{k}^{*}$.

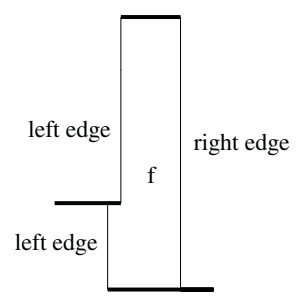

(a)

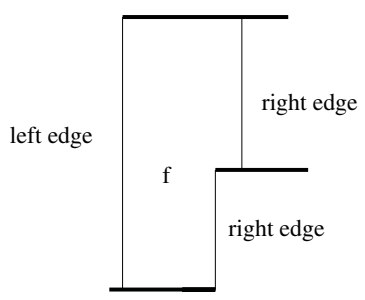

(b)

Fig. 4. (a) Two left edges and one right edge, (b) one left edge and two right edges. 
Proof of Claim: Let $P$ be a longest directed path of $G_{k}^{*}$ in the st-orientation $\xi^{*}$. Obviously, it starts from its source $s^{*}$, i.e. an interior face of $G_{k}$. And it ends at its sink $t^{*}$, the exterior face of $G_{k}$. We try to trace $P$ in $\mathcal{R}_{k}$. It starts from the leftmost interior face. Because $G_{k}$ is a plane triangulation, each face $f$ of $G_{k}$ only has two possible representations in $\mathcal{R}_{k}$ as shown in Figure 4. Its right edges in $\mathcal{R}_{k}$ always have bigger $x$-coordinates than its left edges. Therefore, when $P$ passes through one face of $G_{k}$ (i.e. one vertex in $G_{k}^{*}$ ), it enters a face $f$ from one of its left edges, and it walks out of $f$ from one of its right edges. Thus, each edge on $P$ has to add at least 1 to the $x$-coordinate of the $\operatorname{VR} \mathcal{R}_{k}$. Therefore, the width of $\mathcal{R}_{k}$ is at least the length of $P$. End of the proof of Claim.

Now, we only need to show that for any st-numbering of $G_{k}$, the length of its longest directed paths in its dual st-orientation is no short than $\left\lfloor\frac{4 n}{3}\right\rfloor-3=4 k-3$. We want to show this by induction: When $k=1$, this is trivially true. Assume that it is true when $k=t$. Consider the case of $k=t+1$ : Given any st-numbering $\xi_{t+1}$ of $G_{t+1}$, it induces an $s t$-numbering $\xi_{t}$ of $G_{t}$. According to the induction hypothesis, a longest directed path $P_{t}$ in its dual orientation $\xi_{t}^{*}$ is no shorter than $4 t-3$. Consider $P_{t}$ in $\xi_{t+1}^{*}$, it can be extended to a directed path $P_{t+1}$ in $\xi_{t+1}^{*}$ from its source to its sink. Because of the way $G_{t+1}$ is nested, $P_{t+1}$ has to pass through at least 2 vertices $\left(2\right.$ faces of $G_{t+1}$ ) before it can share edges with the path $P_{t}$. (see Figure 3 ) After it passes through $P_{k}$, it also has to pass through at least 2 vertices ( 2 faces of $G_{t+1}$ ) before it can reach its sink. Therefore, the length of $P_{t+1}$ has to be at least 4 plus the length of $P_{t}$. Therefore, the length of $P_{t+1}$ has to be at least $4(t+1)-3$. This finishes our proof.

\section{References}

1. H. de Fraysseix, J. Pach and R. Pollack, How to draw a planar graph on a grid. Combinatorica 10 (1990), 41-51.

2. G. Kant, A more compact visibility representation. International Journal of Computational Geometry and Applications 7 (1997), 197-210.

3. G. Kant and X. He, Regular edge labeling of 4-connected plane graphs and its applications in graph drawing problems. Theoretical Computer Science 172 (1997), 175-193.

4. A. Lempel, S. Even and I. Cederbaum, An algorithm for planarity testing of graphs, in Theory of Graphs, pp. 215-232, Rome, 1967.

5. C.-C. Lin, H.-I. Lu and I-F. Sun, Improved compact visibility representation of planar graph via Schnyder's realizer, in: Proc. STACS'03, LNCS, Vol. 2607, (SpringerVerlag, Berlin, 2003) 14-25.

6. P. Rosenstiehl and R. E. Tarjan, Rectilinear planar layouts and bipolar orientations of planar graphs. Discrete Comput. Geom. 1 (1986), 343-353.

7. R. Tamassia and I.G.Tollis, An unified approach to visibility representations of planar graphs. Discrete Comput. Geom. 1 (1986), 321-341.

8. H. Zhang and X. He, Compact Visibility Representation and Straight-Line Grid Embedding of Plane Graphs, in: Proc. WADS'03, LNCS, Vol. 2748, (SpringerVerlag Heidelberg, 2003) 493-504.

9. H. Zhang and X. He, On Visibility Representation of Plane Graphs, in: Proc. STACS'04, LNCS, Vol. 2996, (Springer-Verlag Heidelberg, 2004) 477-488. 